\title{
Carbohidratos y proteínas en microalgas: potenciales alimentos funcionales
}

\section{Carbohydrates and proteins in microalgaes: potential functional foods}

\author{
Valeria OImedo Galarza ${ }^{1 *}$ (it) \\ ${ }^{1}$ Universidad Tecnica del Norte (UTN), Ibarra/Imbabura - Ecuador
}

${ }^{*}$ Corresponding Author: Valeria Olmedo Galarza, Universidad Tecnica del Norte (UTN), Av. 17 de Julio, 5-21, y General José María Córdova, 100105, Ibarra/Imbabura - Ecuador, e-mail: volmedo@utn.edu.ec

Cite as: Olmedo Galarza, V. (2019). Carbohydrates and proteins in microalgaes: potential functional foods. Brazilian Journal of Food Technology, 22, e2019043. https://doi.org/10.1590/1981-6723.04319

\begin{abstract}
Resumen
Las microalgas son organismos distribuidos en todo el planeta, con cientos de miles de especies desde que se tienen registros. Sus componentes con potenciales propiedades benéficas en la nutrición y la salud han despertado el interés científico, industrial y comercial. En las últimas décadas se han logrado desarrollar varios productos, entre ellos alimentos funcionales, usando compuestos extraídos de ellas o con la totalidad de su biomasa. Su alta tasa de crecimiento, bajo consumo de nutrientes, relativamente pequeño espacio de producción, plantean un horizonte prometedor como recurso renovable y con grandes beneficios. Las tecnologías para su aprovechamiento se han incrementado y adaptado para mejorar el rendimiento y la calidad, sin embargo, los científicos reconocen que aún existen una serie de desafíos por superar.
\end{abstract}

Palabras clave: Péptidos; $\beta$-glucanos; Compuestos bioactivos; Antioxidantes; Antihipertensivos; Prebióticos; Avances tecnológicos.

\begin{abstract}
Microalgae are organisms distributed throughout the planet, with hundreds of thousands of species since they have records. Its components with potential beneficial properties in nutrition and health have aroused scientific, industrial and commercial interest. In recent decades, several products have been developed, including functional foods and nutraceuticals, using compounds extracted from them or with all their biomass. Its high growth rate, low nutrient consumption, relatively small production space, pose a promising horizon as a renewable resource with great benefits. The technologies for their use have been increased and adapted to improve performance and quality, however, scientists recognize that there are still a number of challenges to be achieved.
\end{abstract}

Keywords: Peptides; $\beta$-glucans; Bioactive compounds; Antioxidants; Antihypertensives; Prebiotics; Technological advances. 


\section{Introducción}

Las microalgas son los organismos más simples del reino vegetal con una gran capacidad de adaptación a distintos medios, lo que ha dado origen a alrededor de 100,000 especies identificadas (Guiry et al., 2014) y distribuidas alrededor del mundo en prácticamente todos los ambientes. Los primeros registros que se tienen acerca de su uso datan de hace 2000 años cuando los chinos utilizaron al género Nostoc sp. como alimento en épocas de hambruna. La siguiente referencia que se tiene de su aplicación en la nutrición es en el tratamiento de pacientes con lepra, a quienes se suplemento su dieta con una sopa que contenía Chlorella sp., observándose un incremento de su peso, energía y salud general. Es después de la segunda guerra mundial cuando se empiezan a cultivar como una solución al déficit de alimentos en Japón y se desarrollan varios productos enriquecidos con microalgas. A partir de entonces la mayor parte de investigaciones se han enfocado en la producción de biomasa, tanto para la obtención de biocombustibles, pero sobre todo como una fuente de alimentos altos en proteína, para humanos y animales, especialmente en acuacultura (Rani et al., 2018; Burja \& Radianingtyas, 2008). Estudios han mostrado que las microalgas pueden representar una fuente atractiva de compuestos con actividad biológica como, por ejemplo, ácidos grasos poliinsaturados, carotenoides, ficobilinas, péptidos y polisacáridos; también son una buena fuente de vitaminas A, B1, B2 y B12. Actualmente se comercializan varios productos en forma de tabletas, polvo, solución o en mezclas con snacks, galletas, fideos, bebidas, caramelos, gomas, vinos y cereales (Sathasivam et al., 2019). Entre los países que van a la vanguardia en la producción se encuentran China, India, Taiwan, Alemania y unos pocos en Latinoamérica. Los géneros que destacan son principalmente Arthospira (comercialmente conocida como Spirulina) y Chlorella, con aproximadamente 12.000 y 5.000 toneladas de producción al año respectivamente y en décadas recientes también se han empezado a estudiar y producir biomasa de los géneros Haematococcus, Dunaliella, Botryococcus, Phaeodactylum, Porphyridium, Chaetoceros, Crypthecodinium, Isochrysis, Nannochloris, Nitzschia, Schizochytrium, Tetraselmis, y Skeletonema. (Rani et al., 2018; Chen et al., 2015; Slocombe et al., 2016; Sathasivam et al., 2019), sin embargo, la cantidad de especies caracterizadas para su potencial aplicación continúa siendo pequeña en relación con la existente y las investigaciones acerca de sus componentes, como aislarlos, las maneras de incrementar su rendimiento, de determinar su bioactividad, sus beneficios o su toxicidad e incluso el desarrollo de productos que sean organolépticamente aceptables son todavía escasas.

\section{Caracterización general de las microalgas}

La palabra alga se encuentra documentada al español en el año 1250 y su significado deriva del griego phykos que significa "hierba de mar". El término es ampliamente utilizado para referirse a todas las plantas que crecen en cuerpos acuáticos, aunque técnicamente no se trata de plantas ya que carecen de raíces, tallos y hojas por lo que más bien pertenecen a las talofitas, es decir que poseen clorofila a como pigmento primario y sus órganos reproductores no están cubiertos (Lee, 2008; Bellinger \& Sigee, 2010) a este amplio grupo pertenecen las microalgas. Se trata de organismos microscópicos, ancestrales, de origen polifilético, es decir que no pertenecen a un solo ancestro común; su tamaño varía entre 20 a $200 \mu \mathrm{m}$, son unicelulares y eucariotas, con excepción de las cianobacterias que son procariotas. Su alimentación es esencialmente fotoautótrofa, es decir que son capaces de utilizar el carbono inorgánico en forma de dióxido de carbono y la energía de la luz del sol para generar moléculas complejas que le sirvan tanto para la formación de sus estructuras como para la generación y reserva de energía (Andersen, 2013b; Barsanti \& Gualtieri, 2006; Falkowski, 2006). Se las puede encontrar en todos los cuerpos acuáticos, en superficies húmedas, en la nieve, sin embargo, Domínguez (2013) menciona que, aunque son ubicuas, la mayoría de las especies se restringen a hábitats específicos, lo que dificulta su completa identificación y descripción.

En la superficie primitiva de la tierra, las microalgas fueron las responsables de producir el oxígeno que cambió la atmosfera y facilitó la evolución de los organismos eucarióticos, de hecho, el petróleo que actualmente se explota, es producto de las macro y microalgas que quedaron enterradas en el Cretácico. 
En nuestros días el 50\% del oxígeno terrestre es gracias a la fotosíntesis realizada por las algas. Además, constituyen el primer eslabón en la cadena trófica para la vida marina y proporcionan una considerable cantidad de compuestos reducidos al medio (Chapman, 2013; Ramanan et al., 2016).

Aunque la estructura celular de las cianobacterias o algas verdeazuladas es relativamente simple y se acerca más a las bacterias, sus 3.500 millones de años de evolución, junto a su gran adaptabilidad, han dado lugar a una amplia diversidad de células, mayor a la que existe entre plantas y animales. La presencia de tilacoides en forma de hojas que ocupan un gran volumen interno origina el pensamiento de que se trate de los progenitores de los cloroplastos en algas y plantas superiores (Andersen, 2013a; Singh \& Saxena, 2015).

Las microalgas eucarióticas, poseen una ultraestructura compleja, su historia de evolución abarca 1.500 millones de años. Exhiben núcleo diferenciado, mitocondrias, uno o más cloroplastos, retículo endoplásmico, sistema de Golgi, junto con otros organelos típicos. Las distintas formas de las eucariotas se muestran no solamente entre especies sino también entre etapas de crecimiento de una misma especie (Lee, 2008; Wehr \& Sheath, 2015). En cuanto a su organización, las microalgas, se presentan como filamentos, formando colonias o como células únicas y a su vez, cada una de estas formas pueden ser inmóviles o móviles mediante flagelos, pseudópodos u oscilaciones (Tomaselli, 2004).

Los cloroplastos son los organelos predominantes en las células eucariotas, que contienen las láminas de tilacoides, en cuyas membranas, se encuentran los pigmentos que captan la luz para la fotosíntesis, haciendo posible la captación del $\mathrm{CO}_{2}$ de la atmósfera para convertirlo en glucosa mediante complejas transformaciones bioquímicas. Las formas de los tilacoides también varían de acuerdo con la especie de microalga, el medio en el cual se desarrolla y la forma en que reserva la energía, por ejemplo, algunos plásmidos contienen pirenoide, que es la acumulación de ribulosa 1,5 bifosfato carboxilasa/oxigenasa, (molécula clave en el ciclo de Calvin de la fotosíntesis). Curiosamente, solo las algas verdes almacenan los productos de la fotosíntesis dentro de sus cloroplastos (Andersen, 2013b; Ball et al., 2011; Singh \& Saxena, 2015), lo que influye en los tipos de pretratamientos que se deben realizar para la extracción de los componentes de interés.

Las vacuolas sirven como reserva de diferentes productos como almidón, polisacáridos de bajo peso molecular o lípidos de los cuales se pueden obtener diversos productos. En cuanto a la pared celular, se trata de una estructura robusta, que permite a la célula aumentar su turgencia sin estallar, la protege y mantiene un equilibrio osmótico con el medio que la rodea permitiendo el paso de materiales mediante un intercambio selectivo que está definido por la composición bioquímica de la membrana. Las microalgas filamentosas y las que forman colonias conectan sus células a través de pasadizos formados en las paredes celulares llamados plasmodesmos (Andersen, 2013b; Singh \& Saxena, 2015).

El aparato de Golgi y el retículo endoplásmico en las microalgas son similares a otras células eucariotas, su función principalmente es segregar productos orgánicos, silicatos, carbonato de calcio, pelos, flagelos y otras estructuras. La composición bioquímica de su pared celular varía entre los grupos, por ejemplo, en las cianobacterias está compuesta por peptidoglicanos en forma de capas asociadas a fibrillas, haciendo de esta capa una rica fuente de proteínas (Andersen, 2013b).

Una forma tradicional de clasificar a las microalgas ha sido de acuerdo con su aspecto citológico, morfológico, los constituyentes de su pared celular y sus pigmentos (Garibay-Hernández et al., 2009; Hu et al., 2008), por ejemplo, las diatomeas marinas deben su color dorado a la presencia de xantofila fucoxantina mientras que las verdeazuladas contienen clorofila a y compuestos relacionados. Sin embargo, el proceso de clasificación es un tema que aún se encuentra en movimiento y gracias a la ayuda de la biología molecular y de la microscopia electrónica se ha logrado circunscribir con mayor precisión a las distintas especies de algas (Barsanti \& Gualtieri, 2006; Domínguez, 2013).

Básicamente pueden reproducirse de forma vegetativa, sexual y asexual. Vegetativa por fisión binaria, asexual cuando forman esporas o sexual cuando existe intercambio genético. Siendo organismos unicelulares su tasa de reproducción y generación de biomasa es mucho mayor que en plantas superiores, requiriendo 
únicamente de luz, macronutrientes en forma de nitrógeno y de carbono inorgánicos y varios minerales como micronutrientes. El carbono que consumen lo fijan para convertirlo en productos de reserva o en moléculas que hacen parte de su estructura formando las biomoléculas (Mondal et al., 2017).

Las proporciones de los componentes bioquímicos varían entre especies y también en una misma especie debido a cambios de $\mathrm{pH}$, salinidad, estrés, temperatura, intensidad de luz, privación de nutrientes, etc. (Hamed, 2016; Duong et al., 2015). Por ejemplo, se incrementa el contenido de lípidos cuando existe déficit de nutrientes como nitrógeno y fósforo en el medio; así también, los aumentos en la intensidad de luz, fuera del rango óptimo requerido por la especie, puede disminuir el contenido de proteínas. Los carotenoides que sirven de protección a la clorofila contra el daño solar se incrementan con el aumento de los rayos UV y de la temperatura, sin embargo, disminuyen cuando se agota el contenido de hierro, estos conocimientos son los que se utilizan actualmente para mejorar el rendimiento del compuesto que se desea producir (Juneja et al., 2013; Sharma et al., 2012).

Los carbohidratos se pueden encontrar en forma de gránulos de almidón como productos de reserva de energía y en otros casos se han hallado segregados por las microalgas en el medio de cultivo. En algunos géneros el polisacárido se presenta con una cadena central de glucosas unidas por enlaces $\alpha(1-4)$ y ramificaciones de cadenas que se unen por enlace $\alpha(1-6)$, es decir más parecido a la subunidad de amilopectina del almidón. Sin embargo, los grados y forma de polimerización de los glucanos es variada, dando origen a productos tales como el paramilón o la crisolaminarina, que se describen como polímeros que presentan diferentes tamaños y tiene en común la unión por enlaces glucosídicos $\beta$, por lo que se conocen como $\beta$-glucanos (Espinoza-Gallardo et al., 2017).

Varios géneros de microalgas son una valiosa fuente de proteínas que compiten favorablemente en cantidad y calidad con productos tradicionales como el huevo, la soya o el pescado. Además, tienen la capacidad de sintetizar todos los 20 aminoácidos y de ser una fuente poco común de los aminoácidos esenciales. Algunos organismos son capaces de secuestrar el nitrógeno, que es variable en la atmósfera para convertirlo en proteínas de reserva que serán utilizadas cuando el nitrógeno escasee (Andersen, 2013b; Espinoza-Gallardo et al., 2017).

Los lípidos han despertado gran interés en la ciencia y la industria debido a su potencial para generar biocombustibles de una fuente renovable, así como por la presencia de ácidos grasos poliinsaturados (PUFAs) que sirvan en la suplementación de alimentos. Sin embargo, las microalgas poseen una diversidad de otros lípidos, mismos que se encuentran formando parte de las membranas, como fosfolípidos, esteroles, lipoproteínas, glucolípidos y galactolípidos; estos últimos son los mayores componentes de las membranas tilacoides. Aquellos lípidos que sirven como reserva de energía se encuentran mayormente como mono, di, triacilgliceroles (acilglicelores) y ácidos grasos libres, formando cuerpos lipídicos o gotas de aceite, que hacen parte de los lípidos neutros solubles en solventes orgánicos, mientras que los fosfolípidos y glucolípidos, pertenecen al grupo de lípidos polares, por lo que son total o parcialmente solubles en agua. La mayor parte de ácidos grasos presentes en las microalgas son insaturados e incluyen a los PUFAs como el ácido araquidónico (AA), Eicosapentanoico (EPA) y Docosahexanoico (DHA). Los géneros como Chaetoceros, Nannochloropsis, Pinguiococcus, Pavlova, Isochrysis entre otras similares, típicamente almacenan lípidos en forma de gotas de aceite, especialmente cuando su reserva de carbohidratos está como crisolaminarina (Khozin-Goldberg et al., 2011; Valenzuela et al., 2015).

Los pigmentos naturales en las microalgas son biomoléculas de vital importancia para su supervivencia ya que cumplen un rol fundamental en el metabolismo fotosintético; debido a su origen como metabolito secundario en la síntesis de lípidos, se clasifica dentro de este grupo. Presentan al menos tres tipos de pigmentos, ficobilinas, carotenoides y clorofilas. Las ficobilinas se hallan como grupos prostéticos formando parte de las ficobiliproteínas que son hidrosolubles y comprenden una gran porción de la proteína total de la célula, por lo que son relativamente fáciles de aislar y purificar; su función es recolectar la luz durante la fotosíntesis. Por otro lado, las clorofilas son pigmentos verdosos liposolubles, que tienen la responsabilidad 
de transformar la energía lumínica en energía química, su porcentaje varía entre un 0,5 a $1 \%$ del peso seco de biomasa. Los carotenoides tienen dos misiones en la fotosíntesis, en primer lugar, deben absorber la luz en regiones del espectro visible donde la clorofila es menos eficiente, debido a esto presentan una gama de colores que van desde el amarillo al marrón; en segundo lugar, actúan como fotoprotectores, absorbiendo el exceso de luz que podría dañar los mecanismos fotosintéticos. De los carotenoides identificados, los más estudiados son $\beta$-caroteno, licopeno, astaxantina y luteína (Cuellar-Bermudez et al., 2015; D'Alessandro \& Antoniosi Filho, 2016; Del Campo et al., 2007).

\section{Carbohidratos y proteínas con actividad biológica}

Varios estudios han mostrado que las biomoléculas presentes en las microalgas tienen la capacidad de modificar la respuesta biológica de las células, lo que ha incrementado el interés por aislarlos y probar tanto sus efectos benéficos, como las dosificaciones y posible toxicidad. Los resultados obtenidos hasta el momento se muestran prometedores para la aplicación en productos, que van desde lo nutricional a lo farmacéutico; a continuación, se recogen las investigaciones recientes en carbohidratos y proteínas, realizadas con la finalidad de determinar sus efectos bioactivos.

La elaboración de productos alimenticios suplementados con microalgas, en primera instancia fue impulsado por el alto contenido de proteínas, de alrededor del 50\%, encontrado en géneros como Chlorella, Scenedesmus, Arthospira, entre otros; además, esta proteína presenta mayor valor nutricional que la encontrada en varios de los cereales y leguminosas consumidos habitualmente, aunque no es superior a la de fuentes animales (Burja \& Radianingtyas, 2008). El mayor porcentaje de productos que se comercializan actualmente son suplementados principalmente con la biomasa seca de microalgas y aunque la mayoría de resultados obtenidos son prometedores, también se han dado casos de efectos secundarios y alergias, que pueden deberse a la estructura tridimensional de las proteínas, a otros componentes presentes en la célula o incluso a un procesamiento insuficiente, dificultando la digestibilidad y ocasionando trastornos intestinales (Rani et al., 2018), lo que indica la necesidad de incrementar esfuerzos para el aislamiento de proteínas en su forma nativa y la determinación de su bioactividad, sin embargo esto se ha dificultado debido a la sensibilidad de las proteínas de microalgas a los procesos de extracción y aislamiento existentes que las desnaturalizan, por lo que las investigaciones mayormente han observado los efectos de los péptidos obtenidos mediante hidrólisis inducidas con enzimas específicas obtenidas de plantas, microorganismos y animales (Ejike et al., 2017), en este sentido se ha observado el número y secuencia de aminoácidos son factores preponderantes, por lo tanto, de acuerdo con las posibles combinaciones se podrían obtener una gran variedad de resultados, sin embargo, hasta el momento se han agrupado en 4 tipos: antioxidante, antihipertensivo, hipolipidemiante y antimicrobial (Yücetepe \& Özçelik, 2016)

La actividad antioxidante se ha convertido en una tendencia no solo en el ámbito de la nutrición, sino además en las investigaciones relacionadas con la industria de los alimentos debido a la intención de reemplazar los antioxidantes sintéticos, como el butil hidroxianizol (BHA) y el butil hidroxitolueno (BHT), debido a su riesgo potencial para la salud, por antioxidantes naturales provenientes de microalgas (Kim \& Kang, 2011). Por otra parte, de acuerdo con los datos de la Organización Mundial de la Salud (2017), el 31\% del total de muertes ocurridas en el 2015, fueron ocasionadas por enfermedades cardiovasculares (ECV), de ellas, aproximadamente la mitad son causadas por la hipertensión que tiene como factor clave de su desarrollo al estrés oxidativo. El aumento de las especies reactivas de oxígeno (ROS) puede causar oxidación de las macromoléculas y disfunción endotelial, lo que convierte al estrés oxidativo en un mediador del desbalance entre los mecanismos vasodilatador y vasoconstrictor, también se asocia con enfermedades crónicas degenerativas como el cáncer, la diabetes y el envejecimiento prematuro. Estos hallazgos han motivado investigaciones y estrategias que además de buscar cambios en los estilos de vida de las personas, también desarrollen productos naturales que puedan otorgar beneficios similares o mejores a los fármacos de tratamiento, pero sin los efectos secundarios de estos (Ejike et al., 2017). 
La investigación in-vitro realizada por Norzagaray-Valenzuela et al. (2017), probó el extracto residual de biomasa de tres especies de microalgas (Dunaliella tertiolecta, Tetraselmis suecica y Nannochloropsis sp.) y mostró alta capacidad antioxidante y potencial antienvejecimiento de los extractos residuales hidrolizados y sin hidrolizar de D. tertiolecta y Nannochloropsis sp.; asi también, usando la especie $N$. oculata, se aislaron y purificaron dos péptidos (tripéptido y heptapéptido) que mostraron actividad antihipertensiva por inhibición de la enzima convertidora de angiotensina I en angiotensina II, que es la responsable de la contracción de los vasos sanguíneos y la primera línea terapéutica contra la hipertensión (Samarakoon et al., 2013). Resultados similares se obtuvieron mediante la purificación e identificación de cuatro péptidos obtenidos de la especie Tetradesmus obliquus, que también fueron testeados in-vitro (Montone et al., 2018) y del extracto hidrolizado de proteína de la especie Scenedesmus obliqus (Afify et al., 2018).

Derivado de la capacidad antioxidante, otra investigación mostró un efecto hepato-protector, al administrar extracto de Arthospira platensis junto con acetato de plomo, observándose una reducción del daño hepático inducido por el plomo, resultado que se atribuye a la capacidad secuestrante de radicales presente en los componentes de la microalga (El-Tantawy, 2015).

El efecto antihipertensivo en animales de ha mostrado mediante la administración de hidrolizado de proteínas obtenido de C. sorokiniana; en la identificación de las diferentes fracciones se destacó un grupo de dipéptidos con aminoácidos hidrofóbicos terminales que mostró una fuerte inhibición de angiotensina I en las pruebas in-vitro y una disminución significativa de la presión arterial en ratones espontáneamente hipertensos frente a los controles (Lin et al., 2018). Investigaciones anteriores ya habían mostrado resultados similares, pero en las especies Chlorella vulgaris, C. ellipsoidea, y A. platensis (Suetsuna \& Chen, 2001; Ko et al., 2012). Si bien, estos péptidos se pueden encontrar en otros alimentos como salmón o krill con los que se suplementan alimentos funcionales para la hipertensión, la opción de usar microalgas resulta más económica y renovable (Lin et al., 2018).

El aparecimiento de cepas patógenas cada vez más resistentes a los antibióticos tradicionales, así como los efectos secundarios de los mismos también ha motivado la investigación para encontrar alternativas, es así como, en el estudio de Sun, etal. (2016) se muestra la capacidad antibacterial de un péptido de 18 aminoácidos obtenido mediante hidrólisis del extracto proteico de A. platensis, frente tanto a bacterias Gram positivas como Gram negativas y específicamente contra E. coli y S. aureus.

La biomasa de los géneros Arthospira y Chlorella ha sido utilizada en varios ensayos clínicos con resultados que incluyen la disminución de la presión arterial, de los niveles en lípidos totales, en colesterol y LDL, efecto inmunoestimulante, modulación del estrés oxidativo y decremento de los niveles de glucosa (Lee et al., 2008; Torres-Durán et al., 2007, 2012; Kwak et al., 2012; Panahi et al., 2013; Ngo-Matip et al., 2015; Alam et al., 2016). Las investigaciones han mostrado el potencial de las microalgas, con la limitante de no usarlo en pacientes con insuficiencia renal debido al alto contenido de proteínas y fósforo. Por otra parte, cabe mencionar que, debido a la administración de la biomasa total, los resultados no son atribuibles a un componente en particular de las microalgas y se plantea la incógnita de saber si es la sinergia de las biomoléculas la que arroja tales resultados o si estos podrían ser mejores y/o tener menos restricciones al utilizar elementos aislados.

En el grupo de los carbohidratos se destacan los $\beta$-glucanos, de los cuales, los más conocidos son el paramilón, presente en euglenoides y la crisolaminarina, hallada en las diatomeas. El paramilón es un polisacárido lineal, no ramificado, formado por la unión de D-glucosas mediante enlaces $\beta 1-3$ y su estructura tridimensional es helicoidal, mientras que la crisolaminarina presenta la misma cadena principal con ramificaciones $\beta 1-6$ (Espinoza-Gallardo et al., 2017; Nakashima et al., 2018).

Estudios in vitro realizados por Ujita et al. (2009) mostraron un efecto inmuno-estimulador debido a la unión de los $\beta$-glucanos con receptores específicos, denominados Dectina-1, presentes en la superficie de los macrófagos. Más aún, fracciones purificadas y solubles de $\beta$-(1,3)(1,6)-glucano administradas de forma oral en ratones, mostraron efectos de inhibición al crecimiento de tumores, por lo que sugieren una modificación 
positiva a la respuesta inmune de las células T (Masuda et al., 2012, 2013). Jesus Raposo et al. (2015) resume similares resultados encontrados al utilizar diversas especies de microalgas que presentan considerables cantidades de $\beta$-glucanos. Otros polisacáridos tales como $\mathrm{N}$-acetil-glucosamina y $\mathrm{N}$-acetil-galactosamina, presentes en el extracto de Chlorella pyrenoidosa y Chlorella ellipsoidea fueron probados por Hasegawa et al. (1995 apud Rani et al., 2018), mostrando acción inhibitoria contra Listeria monocytogenes y el hongo Candida albicans.

Una acción antialérgica del paramilón, también fue testeada mediante la administración oral de este, en ratones con síntomas de dermatitis atópica, mostrando disminución de los síntomas de esta enfermedad y aunque el estudio requiera de mayores evidencias, para escalar al uso en humanos, proporciona una potencial alternativa al uso de fármacos esteroideos (Sugiyama et al., 2010). Otro estudio in vitro realizado por Casas-Arrojo et al. (2018), mostró resultados positivos en actividad inmunomoduladora, antioxidante y anticancerígena de polisacáridos extraídos de Euglena gracilis, lo que sugiere considerar el uso de estos polisacáridos como potenciales nutracéuticos.

Por otra parte, el alto contenido de polisacáridos y oligosacáridos en forma de fibra soluble e insoluble de algunas especies de microalgas es la propiedad que se atribuye a su actividad prebiótica, que por sí misma promueve el balance adecuado de la microbionta intestinal y esto a su vez gatilla una serie de efectos bioquímicos y fisiológicos en el huésped que mejoran su metabolismo (Jesus Raposo et al., 2016). Tal es el caso de la investigación realizada por Beheshtipour et al. (2012) en la que el uso de la biomasa de Arthrospira platensis y Chlorella vulgaris incrementaron la viabilidad de las bacterias probióticas Lactobacilus acidophilus y Bifidobacterium lactis. Otra investigación enfocada en este mismo efecto prebiótico utilizó la biomasa de Isochrysis galbana en modelos de ratones diabéticos, observándose una disminución de sus niveles de glucosa y colesterol, manteniendo estable su peso corporal (Nuño et al., 2013), lo que sugiere su uso como suplemento coadyuvante en el control de los niveles de glucosa para personas con diabetes o como un alimento, que consumido regularmente podría servir para prevenir la enfermedad.

\section{Tecnologías actuales de extracción e identificación}

Entre las ventajas del cultivo de las microalgas se cuentan que se puede llevar a cabo independientemente del suministro de agua dulce y no compite con tierra cultivable o paisajes biodiversos. De hecho, muchas microalgas con beneficios para la salud son marinas o salobres. Por lo tanto, las microalgas se consideran una fuente ideal para la producción sostenible de compuestos fisiológicamente activos, sin embargo, una de las principales dificultades existentes en el desarrollo actual de la industria de microalgas se debe a que la mayoría de técnicas seleccionadas, se hacen en base al tipo de metabolito que se quiere obtener y no a la producción industrial, el rendimiento y el mercado objetivo (Ejike et al., 2017), es así que los resultados a nivel de laboratorio suelen ser exitosos, pero una vez que se quiere escalar a la industria es necesario poner mayor énfasis en el control de posibles contaminaciones, por ejemplo con metales pesados o con especies distintas que en algunos casos pueden ser tóxicas.

Una vez determinadas las condiciones óptimas de crecimiento, recolección y secado de la biomasa, de acuerdo con la especie seleccionada, se presenta el desafío de extraer sus metabolitos mediante un pretratamiento que permita la ruptura de la pared celular, que en algunos casos puede soportar presiones de hasta 9,5 MPa, convirtiendo a este paso en decisivo para obtener un buen rendimiento (Phong et al., 2018). Con este objetivo se utilizan varias técnicas, tal como recogen las investigaciones de Lee et al. (2010), Onay et al. (2016) y Suarez-García et al. (2018) en las que se menciona el uso de la autoclave, microondas, sonicador, shock osmótico con solución salina, fresado con cuentas de zirconio o cristal, homogenización sobre hielo o la liofilización. Por otra parte, el uso de enzimas permite mejorar la extracción de proteínas en algunas especies de microalgas, tal como lo indica la investigación realizada por Al-Zuhair et al. (2017), aunque el uso de fresado con cuentas es el método preferido a nivel industrial gracias a la posibilidad de usar grandes cargas de biomasa y la alta eficiencia de disrupción. Otras tecnologías se encuentran aún en desarrollo 
como son la micro fluidización, tecnología de arco pulsado, membranas cubiertas de polímero catiónico o la descompresión explosiva, que entre sus ventajas se cuentan el uso de temperaturas medias, que no necesitan usar solventes o ser amigables con el ambiente. En general, la selección del método de disrupción celular se realiza con base en la facilidad de aplicación, la menor contaminación de metabolitos, la más baja obtención de desechos celulares así como la disminución de costos y energía (Suarez-García et al., 2018; Phong et al., 2018).

Cuando se ha realizado la lisis celular se procede con sucesivas operaciones de separación y purificación. El uso de la centrifugación diferencial permite precipitar las proteínas de membrana de las proteínas solubles, que quedan en el sobrenadante. Luego los extractos pueden someterse a procesos de fraccionamiento, donde las proteínas pueden ser purificadas de acuerdo con sus propiedades de solubilidad, carga y tamaño (Cavonius et al., 2015; Slocombe et al., 2013).

La precipitación salina permite la separación y purificación de proteínas particulares usando sus diferencias de solubilidad a distintas concentraciones de sales. El sulfato de amonio suele ser la más utilizada debido a su alta solubilidad $\left(760 \mathrm{~g}\right.$ por litro a $\left.20^{\circ} \mathrm{C}\right)$. Una de las ventajas de esta técnica es que la adición gradual de la sal separa las proteínas sin desnaturalizarlas, lo que permite aprovechar su actividad biológica.

La extracción de carbohidratos se puede hacer mediante ácido sulfúrico o el uso de enzimas, mientras que su identificación y cuantificación se realiza tradicionalmente mediante espectrofotometría con la conversión a furanos mediante el ácido fenol-sulfúrico, sin embargo, este método es poco selectivo por lo que se corre el riesgo de desestimar o sobreestimar el contenido real de carbohidratos. El estudio de Templeton et al. (2012) muestra una mejor separación e identificación de los carbohidratos de microalgas con el uso de la cromatografía líquida de alta resolución (HPLC) por intercambio iónico, frente a la cromatografía de gases.

El uso de HPLC en sus distintas columnas por diferencia de tamaño, intercambio iónico o aún más específica de antígeno anticuerpo, son técnicas que permiten la separación e identificación tanto de proteínas como de carbohidratos de manera eficiente.

\section{Conclusión}

La creciente investigación en el campo de las microalgas evidencia el potencial que tienen en diferentes áreas, sin embargo, el mayor interés e inversión que se está realizando en este campo se enfoca en la obtención de biocombustibles (biodiesel y bioalcohol) lo que de alguna manera retrasa los avances en la obtención de otros metabolitos para uso alimenticio y farmacéutico. Es así que las tecnologías actuales aún requieren mejoras que permitan obtener metabolitos sin contaminación y determinar sus propiedades y actividades biológicas. Por otra parte es necesario desarrollar alimentos que además de nutritivos y con características funcionales sean aceptados de manera masiva por los consumidores, sin que los costos sean elevados.

\section{Referencias}

Afify, A. E.-M. M. R., El Baroty, G. S., El Baz, F. K., Abd El Baky, H. H. E., \& Murad, S. A. (2018). Scenedesmus obliquus: Antioxidant and antiviral activity of proteins hydrolyzed by three enzymes. Journal of Genetic Engineering and Biotechnology, 16(2), 399-408. PMid:30733753. http://dx.doi.org/10.1016/j.jgeb.2018.01.002

Alam, A., Quamri, S., Fatima, S., Roqaiya, M., \& Ahmad, Z. (2016). Efficacy of Spirulina (Tahlab) in patients of type 2 diabetes mellitus (Ziabetus Shakri): A randomized controlled trial. Journal of Diabetes \& Metabolism, 7(10), 1-5. http://dx.doi.org/10.4172/2155-6156.1000710

Al-Zuhair, S., Ashraf, S., Hisaindee, S., Darmaki, N. A., Battah, S., Svistunenko, D., Reeder, B., Stanway, G., \& Chaudhary, A. (2017). Enzymatic pre-treatment of microalgae cells for enhanced extraction of proteins. Engineering in Life Sciences, 17(2), 175-185. http://dx.doi.org/10.1002/elsc.201600127

Andersen, R. A. (2013a). Advances in algal cell biology. Phycologia, 52(4), 383-384. http://dx.doi.org/10.2216/13-BR4.1

Andersen, R. A. (2013b). The microalgal cell. In A. Richmond \& Q. Hu (Eds.), Handbook of microalgal culture (Chap. 1, 1st ed., pp. 1-20). Oxford: John Wiley \& Sons. http://dx.doi.org/10.1002/9781118567166.ch1.

Ball, S., Colleoni, C., Cenci, U., Raj, J. N., \& Tirtiaux, C. (2011). The evolution of glycogen and starch metabolism in eukaryotes gives molecular clues to understand the establishment of plastid endosymbiosis. Journal of Experimental Botany, 62(6), 17751801. PMid:21220783. http://dx.doi.org/10.1093/jxb/erq411 
Barsanti, L., \& Gualtieri, P. (2006). General overview. In L. Barsanti \& P. Gualtieri (Eds.), Algae: Anatomy, biochemistry, and biotechnology (Chap. 1, pp. 1-44). Boca Ratón: CRC Press.

Beheshtipour, H., Mortazavian, A. M., Haratian, P., \& Darani, K. K. (2012). Effects of Chlorella vulgaris and Arthrospira platensis addition on viability of probiotic bacteria in yogurt and its biochemical properties. European Food Research and Technology, 235(4), 719-728. http://dx.doi.org/10.1007/s00217-012-1798-4

Bellinger, E. G., \& Sigee, D. C. (2010). Introduction to freshwater algae. In E. G. Bellinger \& D. C. Sigee (Eds.), Freshwater algae: Identification and use as bioindicators (Chap. 1, pp. 1-40). New Delhi: Wiley-Blackwell. http://dx.doi.org/10.1002/9780470689554.ch1.

Burja, A. M., \& Radianingtyas, H. (2008). Marine microbes: An introduction to a diverse group of natural products isolated from marine macroalgae, microalgae, bacteria, fungi, and cianobacteria. In C. Barrow \& F. Shahidi (Eds.), Marine nutraceuticals and functional foods (Chap. 15, pp. 367-403). Boca Ratón: Taylor \& Francis.

Casas-Arrojo, V., Ortega-Rodríguez, A., Arrojo-Agudo, M. A., \& Abdala-Díaz, R. T. (2018). Immunomodulatory, antioxidant and anti-tumor capacity of acidic polysaccharides from Euglena gracilis. In Libro de Resúmenes del VI Congreso Latinoamericano de Biotecnología Algal. Lima: Universidad Científica del Sur. Recuperado el 17 de septiembre de 2019, de https://www.academia.edu/35318630/EVALUACI\%C3\%93N_DEL_FOTOPERIODO_EN_EL_ASENTAMIENTO_DE_TETRAES PORAS_DE_CHONDRACANTHUS_CHAMISSOI_SOBRE_CUERDAS_DE_POLIPROPILEN̄̄O_EN_CONDICIONES_SEMICONTROLADAS_DE_LABORATORIO

Cavonius, L. R., Albers, E., \& Undeland, I. (2015). pH-shift processing of Nannochloropsis oculata microalgal biomass to obtain a protein-enriched food or feed ingredient. Algal Research, 11, 95-102. http://dx.doi.org/10.1016/j.algal.2015.05.022

Chapman, R. L. (2013). Algae: The world's most important 'plants': An introduction. Mitigation and Adaptation Strategies for Global Change, 18(1), 5-12. http://dx.doi.org/10.1007/s11027-010-9255-9

Chen, J., Wang, Y., Benemann, J. R., Zhang, X. H. U. H., \& Qin, S. (2015). Microalgal industry in China: Challenges and prospects. In Proceedings of the 1st International Coastal Biology Congress. Yantai: Springer. Recuperado el 13 de agosto de 2018, de https://www.researchgate.net/profile/Jun_Chen78/publication/282882417_Microalgal_industry_in_China_challenges_ and_prospects/links/5621a44908ae70315b58cfa2.pdf

Cuellar-Bermudez, S. P., Aguilar-Hernandez, I., Cardenas-Chavez, D. L., Ornelas-Soto, N., Romero-Ogawa, M. A., \& ParraSaldivar, R. (2015). Extraction and purification of high-value metabolites from microalgae: Essential lipids, astaxanthin and phycobiliproteins. Microbial Biotechnology, 8(2), 190-209. PMid:25223877. http://dx.doi.org/10.1111/1751-7915.12167

D'Alessandro, E. B., \& Antoniosi Filho, N. R. (2016). Concepts and studies on lipid and pigments of microalgae: A review. Renewable \& Sustainable Energy Reviews, 58, 832-841. http://dx.doi.org/10.1016/j.rser.2015.12.162

Del Campo, J. A., García-González, M., \& Guerrero, M. G. (2007). Outdoor cultivation of microalgae for carotenoid production: Current state and perspectives. Applied Microbiology and Biotechnology, 74(6), 1163-1174. PMid:17277962. http://dx.doi.org/10.1007/s00253-007-0844-9

Domínguez, H. (2013). Algae as a source of biologically active ingredients for the formulation of functional foods and nutraceuticals. In H. Domínguez, Functional ingredients from algae for foods and nutraceuticals (pp. 1-86). Vigo: Elsevier Science. http://dx.doi.org/10.1533/9780857098689.1.

Duong, V. T., Thomas-Hall, S. R., \& Schenk, P. M. (2015). Growth and lipid accumulation of microalgae from fluctuating brackish and sea water locations in South East Queensland-Australia. Frontiers of Plant Science, 6, 359. PMid:26042142. http://dx.doi.org/10.3389/fpls.2015.00359

Ejike, C. E. C. C., Collins, S. A., Balasuriya, N., Swanson, A. K., Mason, B., \& Udenigwe, C. C. (2017). Prospects of microalgae proteins in producing peptide-based functional foods for promoting cardiovascular health. Trends in Food Science \& Technology, 59, 30-36. http://dx.doi.org/10.1016/j.tifs.2016.10.026

El-Tantawy, W. H. (2015). Antioxidant effects of Spirulina supplement against lead acetate-induced hepatic injury in rats. Journal of Traditional and Complementary Medicine, 6(4), 327-331. PMid:27774414. http://dx.doi.org/10.1016/j.jtcme.2015.02.001

Espinoza-Gallardo, D., Contreras-Porcia, L., \& Ehrenfeld, N. (2017). B-glucanos, su producción y propiedades en microalgas con énfasis en el género Nannochloropsis (Ochrophyta, Eustigmatales). Revista de Biología Marina y Oceanografía, 52(1), $33-$ 49. http://dx.doi.org/10.4067/S0718-19572017000100003

Falkowski, P. G. (2006). Evolution: Tracing oxygen's imprint on earth's metabolic evolution. Science, 311(5768), 1724-1725. PMid:16556831. http://dx.doi.org/10.1126/science.1125937

Garibay-Hernández, A., Vázquez-Duhalt, R., Sánchez Saavedra, M., Serrano Carreón, L., \& Martínez Jiménez, L. (2009). Biodiesel a partir de microalgas. BioTecnología, 13(3), 38-61.

Guiry, M. D., Guiry, G. M., Morrison, L., Rindi, F., Miranda, S. V., Mathieson, A. C., Parker, B. C., Langangen, A., John, D. M., Bárbara, I., Carter, C. F., Kuipers, P., \& Garbary, D. J. (2014). AlgaeBase: An on-line resource for algae. Cryptogamie, 35(2), 105-115. http://dx.doi.org/10.7872/crya.v35.iss2.2014.105

Hamed, I. (2016). The evolution and versatility of microalgal biotechnology: A review. Comprehensive Reviews in Food Science and Food Safety, 15(6), 1104-1123. http://dx.doi.org/10.1111/1541-4337.12227

Hu, Q., Sommerfeld, M., Jarvis, E., Ghirardi, M., Posewitz, M., Seibert, M., \& Darzins, A. (2008). Microalgal triacylglycerols as feedstocks for biofuel production: Perspectives and advances. The Plant Journal, 54(4), 621-639. PMid:18476868. http://dx.doi.org/10.1111/j.1365-313X.2008.03492.x

Jesus Raposo, M. F., Morais, R. M. S. C., \& Morais, A. M. M. B. (2015). Marine polysaccharides from algae with potential biomedical applications. Marine Drugs, 13(5), 2967-3028. PMid:25988519. http://dx.doi.org/10.3390/md13052967 
Jesus Raposo, M. F., Morais, R. M. S. C., \& Morais, A. M. M. B. (2016). Emergent sources of prebiotics: Seaweeds and Microalgae. Marine Drugs, 14(2), 1-27. PMid:26828501. http://dx.doi.org/10.3390/md14020027

Juneja, A., Ceballos, R., \& Murthy, G. (2013). Effects of environmental factors and nutrient availability on the biochemical composition of algae for biofuels production: A review. Energies, 6(9), 4607-4638. http://dx.doi.org/10.3390/en6094607

Khozin-Goldberg, I., Iskandarov, U., \& Cohen, Z. (2011). LC-PUFA from photosynthetic microalgae: Occurrence, biosynthesis, and prospects in biotechnology. Applied Microbiology and Biotechnology, 91(4), 905-915. PMid:21720821.

http://dx.doi.org/10.1007/s00253-011-3441-x

Kim, S. K., \& Kang, K. H. (2011). Medicinal effects of peptides from marine microalgae. In S. K. Kim (Ed.), Marine medicinal foods: Implications and applications, macro and microalgae (Chap. 25, pp. 313-323). San Diego: Academic Press. http://dx.doi.org/10.1016/B978-0-12-387669-0.00025-9.

Ko, S.-C., Kang, N., Kim, E.-A., Kang, M. C., Lee, S.-H., Kang, S.-M., Lee, J.-B., Jeon, B.-T., Kim, S.-K., Park, S.-J., Park, P.-J., Jung, W.-K., Kim, D., \& Jeon, Y.-J. (2012). A novel angiotensin I-converting enzyme (ACE) inhibitory peptide from a marine Chlorella ellipsoidea and its antihypertensive effect in spontaneously hypertensive rats. Process Biochemistry, 47(12), 20052011. http://dx.doi.org/10.1016/j.procbio.2012.07.015

Kwak, J. H., Baek, S. H., Woo, Y., Han, J. K., Kim, B. G., Kim, O. Y., \& Lee, J. H. (2012). Beneficial immunostimulatory effect of short-term Chlorella supplementation: Enhancement of Natural Killer cell activity and early inflammatory response (Randomized, double-blinded, placebo-controlled trial). Nutrition Journal, 11(1), 53. PMid:22849818. http://dx.doi.org/10.1186/1475-2891-1153

Lee, E. H., Park, J.-E., Choi, Y.-J., Huh, K.-B., \& Kim, W.-Y. (2008). A randomized study to establish the effects of spirulina in type 2 diabetes mellitus patients. Nutrition Research and Practice, 2(4), 295-300. PMid:20016733. http://dx.doi.org/10.4162/nrp.2008.2.4.295

Lee, J.-Y., Yoo, C., Jun, S.-Y., Ahn, C.-Y., \& Oh, H.-M. (2010). Comparison of several methods for effective lipid extraction from microalgae. Bioresource Technology, 101(1, Suppl 1), S75-S77. PMid:19386486.

http://dx.doi.org/10.1016/j.biortech.2009.03.058

Lee, R. E. (2008). Basic characteristics of the algae. In R. E. Lee (Ed.), Phycology (Chap. 1, 4th ed., pp. 3-29). Colorado: Cambridge University Press. http://dx.doi.org/10.1017/CBO9780511812897.002

Lin, Y.-H., Chen, G.-W., Yeh, C., Song, H., \& Tsai, J.-S. (2018). Purification and identification of angiotensin I-converting enzyme inhibitory peptides and the antihypertensive effect of Chlorella sorokiniana protein hydrolysates. Nutrients, 10(10), 1397. http://dx.doi.org/10.3390/nu10101397

Masuda, Y., Inoue, H., Ohta, H., Miyake, A., Konishi, M., \& Nanba, H. (2013). Oral administration of soluble $\beta$-glucans extracted from Grifola frondosa induces systemic antitumor immune response and decreases immunosuppression in tumor-bearing mice. International Journal of Cancer, 133(1), 108-119. PMid:23280601. http://dx.doi.org/10.1002/ijc.27999

Masuda, Y., Togo, T., Mizuno, S., Konishi, M., \& Nanba, H. (2012). Soluble -glucan from Grifola frondosa induces proliferation and Dectin-1/Syk signaling in resident macrophages via the GM-CSF autocrine pathway. Journal of Leukocyte Biology, 91(4), 547-556. PMid:22028332. http://dx.doi.org/10.1189/jlb.0711386

Mondal, M., Goswami, S., Ghosh, A., Oinam, G., Tiwari, O. N., Das, P., Gayen, K., Mandal, M. K., \& Halder, G. N. (2017) Production of biodiesel from microalgae through biological carbon capture: A review. 3 Biotech, 7(2), 99. PMid:28560639. http://dx.doi.org/10.1007/s13205-017-0727-4

Montone, C. M., Capriotti, A. L., Cavaliere, C., La Barbera, G., Piovesana, S., Zenezini Chiozzi, R. E., \& Laganà, A. (2018). Peptidomic strategy for purification and identification of potential ACE-inhibitory and antioxidant peptides in Tetradesmus obliquus microalgae. Analytical and Bioanalytical Chemistry, 410(15), 3573-3586. PMid:29476230. http://dx.doi.org/10.1007/s00216-018-0925-x

Nakashima, A., Yamada, K., Iwata, O., Sugimoto, R., Atsuji, K., Ogawa, T., Ishibashi-Ohgo, N., \& Suzuki, K. (2018). $\beta$-glucan in foods and its physiological functions. Journal of Nutritional Science and Vitaminology, 64(1), 8-17. PMid:29491277. http://dx.doi.org/10.3177/jnsv.64.8

Ngo-Matip, M.-E., Pieme, C.-A., Azabji-Kenfack, M., Moukette, B.-M., Korosky, E., Stefanini, P., Ngogang, J.-Y., \& Mbofung, C. M. (2015). Impact of daily supplementation of Spirulina platensis on the immune system of naïve HIV-1 patients in Cameroon: A 12-months single blind, randomized, multicenter trial. Nutrition Journal, 14(1), 70. PMid:26195001. http://dx.doi.org/10.1186/s12937-015-0058-4

Norzagaray-Valenzuela, C. D., Valdez-Ortiz, A., Shelton, L. M., Jiménez-Edeza, M., Rivera-López, J., Valdez-Flores, M. A., \& Germán-Báez, L. J. (2017). Residual biomasses and protein hydrolysates of three green microalgae species exhibit antioxidant and anti-aging activity. Journal of Applied Phycology, 29(1), 189-198. http://dx.doi.org/10.1007/s10811-016-0938-9

Nuño, K., Villarruel-López, A., Puebla-Pérez, A. M., Romero-Velarde, E., Puebla-Mora, A. G., \& Ascencio, F. (2013). Effects of the marine microalgae Isochrysis galbana and Nannochloropsis oculata in diabetic rats. Journal of Functional Foods, 5(1), 106115. http://dx.doi.org/10.1016/j.jff.2012.08.011

Onay, M., Sonmez, C., Oktem, H. A., \& Yucel, M. (2016). Evaluation of various extraction techniques for efficient lipid recovery from thermo-resistant microalgae, Hindakia, Scenedesmus and Micractinium species. American Journal of Analytical Chemistry, 7(02), 141-150. http://dx.doi.org/10.4236/ajac.2016.72012

Organización Mundial de la Salud - OMS. (2017). Monitoreo de avances en materia de las enfermedades no transmisibles 2017. Ginebra: OMS Press. Recuperado el 23 de agosto de 2018, de http://apps.who.int/iris/bitstream/handle/10665/259806/9789243513027-spa.pdf?sequence=1 
Panahi, Y., Mostafazadeh, B., Abrishami, A., Saadat, A., Beiraghdar, F., Tavana, S., Pishgoo, B., Parvin, S., \& Sahebkar, A (2013). Investigation of the effects of Chlorella vulgaris supplementation on the modulation of oxidative stress in apparently healthy smokers. Clinical Laboratory, 59(5-6), 579-587. PMid:23865357. http://dx.doi.org/10.7754/Clin.Lab.2012.120110

Phong, W. N., Show, P. L., Ling, T. C., Juan, J. C., Ng, E.-P., \& Chang, J.-S. (2018). Mild cell disruption methods for biofunctional proteins recovery from microalgae: Recent developments and future perspectives. Algal Research, 31, 506-516. http://dx.doi.org/10.1016/j.algal.2017.04.005

Ramanan, R., Kim, B.-H., Cho, D.-H., Oh, H.-M., \& Kim, H.-S. (2016). Algae-bacteria interactions: Evolution, ecology and emerging applications. Biotechnology Advances, 34(1), 14-29. PMid:26657897. http://dx.doi.org/10.1016/j.biotechadv.2015.12.003

Rani, K., Sandal, N., \& Sahoo, P. K. (2018). A comprehensive review on chlorella-its composition, health benefits, market and regulatory scenario. The Pharma Innovation Journal, 7(7), 584-589.

Samarakoon, K. W., O-Nam, K., Ko, J.-Y., Lee, J.-H., Kang, M.-C., Kim, D., Lee, J. B., Lee, J.-S., \& Jeon, Y.-J. (2013) Purification and identification of novel angiotensin-I converting enzyme (ACE) inhibitory peptides from cultured marine microalgae (Nannochloropsis oculata) protein hydrolysate. Journal of Applied Phycology, 25(5), 1595-1606. http://dx.doi.org/10.1007/s10811-013-9994-6

Sathasivam, R., Radhakrishnan, R., Hashem, A., \& Abd Allah, E. F. (2019). Microalgae metabolites: A rich source for food and medicine. Saudi Journal of Biological Sciences, 26(4), 709-722. PMid:31048995. http://dx.doi.org/10.1016/j.sjbs.2017.11.003

Sharma, K. K., Schuhmann, H., \& Schenk, P. M. (2012). High lipid induction in microalgae for biodiesel production. Energies, 5(5), 1532-1553. http://dx.doi.org/10.3390/en5051532

Singh, J., \& Saxena, R. C. (2015). An introduction to microalgae: Diversity and significance. In S. K. Kim (Ed.), Handbook of marine microalgae (Chap. 2, pp. 11-24). Busan: Elsevier. http://dx.doi.org/10.1016/B978-0-12-800776-1.00002-9.

Slocombe, S. P., Ross, M., Thomas, N., McNeill, S., \& Stanley, M. S. (2013). A rapid and general method for measurement of protein in micro-algal biomass. Bioresource Technology, 129, 51-57. PMid:23232222. http://dx.doi.org/10.1016/j.biortech.2012.10.163

Slocombe, S. P., Zhang, Q. Y., Ross, M., Stanley, M. S., \& Day, J. G. (2016). Screening and Improvement of marine microalgae for oil production. In S. P. Slocombe \& J. R. Benemann (Eds.), Microalgal production for biomass and high-value products (Chap. 4, pp. 91-113). Boca Ratón: CRC Press.

Suarez García, E., Van Leeuwen, J., Safi, C., Sijtsma, L., Eppink, M. H. M., Wijffels, R. H. E., \& Van den Berg, C. (2018). Selective and energy efficient extraction of functional proteins from microalgae for food applications. Bioresource Technology, 268, 197-203. PMid:30077880. http://dx.doi.org/10.1016/j.biortech.2018.07.131

Suetsuna, K. E., \& Chen, J. R. (2001). Identification of antihypertensive peptides from peptic digest of two microalgae, Chlorella vulgaris and Spirulina platensis. Marine Biotechnology, 3(4), 305-309. PMid:14961345. http://dx.doi.org/10.1007/s10126-0010012-7

Sugiyama, A., Hata, S., Suzuki, K., Yoshida, E., Nakano, R., Mitra, S., Arashida, R., Asayama, Y., Yabuta, Y. E., \& Takeuchi, T. (2010). Oral administration of paramylon, a $\beta-1,3-D$-glucan isolated from Euglena gracilis $\mathrm{z}$ inhibits development of atopic dermatitis-like skin lesions in NC/NGA mice. Journal of Veterinary Medical Science, 72(6), 755-763. PMid:20160419.

Sun, Y., Chang, R., Li, Q. E. L. I., \& Li, B. (2016). B. Isolation and characterization of an antibacterial peptide from protein hydrolysates of Spirulina platensis. European Food Research and Technology, 242(5), 685-692. http://dx.doi.org/10.1007/s00217-015-2576-x

Templeton, D. W., Quinn, M., Van Wychen, S., Hyman, D., \& Laurens, L. M. L. (2012). Separation and quantification of microalgal carbohydrates. Journal of Chromatography A, 1270, 225-234. http://dx.doi.org/10.1016/j.chroma.2012.10.034 Tomaselli, L. (2004). The microalgal cell. In A. Richmond, Handbook of microalgal culture (Chap. 1, pp. 3-19). lowa: Blackwell Science.

Torres-Durán, P. V., Ferreira-Hermosillo, A., \& Juarez-Oropeza, M. A. (2007). Antihyperlipemic and antihypertensive effects of Spirulina maxima in an open sample of Mexican population: A preliminary report. Lipids in Health and Disease, 6(1), 33. PMid:18039384. http://dx.doi.org/10.1186/1476-511X-6-33

Torres-Durán, P. V., Ferreira-Hermosillo, A., Ramos-Jiménez, A., Hernández-Torres, R. P., \& Juárez-Oropeza, M. A. (2012). Effect of Spirulina maxima on postprandial lipemia in young runners: A preliminary report. Journal of Medicinal Food, 15(8), 753757. PMid:22738038. http://dx.doi.org/10.1089/jmf.2011.0309

Ujita, M., Nagayama, H., Kanie, S., Koike, S., Ikeyama, Y., Ozaki, T., \& Okumura, H. (2009). Carbohydrate binding specificity of recombinant human macrophage $\beta$-glucan receptor dectin-1. Bioscience, Biotechnology, and Biochemistry, 73(1), 237-240. PMid:19129647. http://dx.doi.org/10.1271/bbb.80503

Valenzuela, A., Sanhueza, J., \& Valenzuela, R. (2015). Las microalgas: Una fuente renovable para la obtención de ácidos grasos omega-3 de cadena larga para la nutrición humana y animal. Revista Chilena de Nutrición, 42(3), 306-310. http://dx.doi.org/10.4067/S0717-75182015000300013

Wehr, J. D., \& Sheath, R. G. (2015). Introduction to freshwater algae. In J. D. Wehr, R. G. Sheath \& J. P. Kociolek (Eds.). Freshwater algae of North America: Ecology and classification (Chap. 1, 2nd ed., p. 1-10). New York: Elsevier. http://dx.doi.org/10.1016/B978-0-12-385876-4.00002-5.

Yücetepe, A., \& Özçelik, B. (2016). Bioactive peptides isolated from microalgae Spirulina platensis and their biofunctional activities. Akademik Gida, 14(4), 412-417. 
Carbohidratos y proteínas en microalgas: potenciales alimentos funcionales

Olmedo Galarza, V.

Financiamiento: Ninguno.

Received: Feb. 25, 2019; Accepted: June 06, 2019 